\title{
Screening of COVID-19 Using Rapid Test Before Gastrointestinal Procedures; Experience in a Limited Resource Hospital
}

\author{
Fauzi Yusuf $^{1 *}$, Desi Maghfirah ${ }^{1}$ and Muhsin Muhsin ${ }^{2}$ \\ ${ }^{1}$ Gastroenterohepatology Division, Department of Internal Medicine Faculty of Medicine, Universitas Syiah Kuala, Banda Aceh, \\ Indonesia \\ ${ }^{2}$ Residency Program at Department of Internal Medicine Faculty of Medicine, Universitas Syiah Kuala, Banda Aceh, Indonesia
}

Submission: October 2020; Revised: October 2020; Accepted: November 2020

*Corresponding author: Fauzi Yusuf; e-mail: fauziyusuf63@gmail.com; tel.: -

\begin{abstract}
Corona Virus Disease 2019 (COVID-19) is an emerging disease announced by the World Health Organization (WHO) as a pandemic disease since March 2020. Several international guidelines suggest using reverse transcriptase polymerase chain reaction (RT-PCR) test as a screening tool before starting gastrointestinal (GI) procedures. Although RT-PCR is a gold standard, it has a limitation as it is expensive and requires special expertise and difficult to implement in limited resource hospitals. Due to lack of RT-PCR tests available and following Indonesian government regulation, thirty patients in a tertiary referred hospital in Banda Aceh were screened with rapid test antigen and antibody for COVID-19 before underwent emergency and urgency GI procedures such as endoscopy, colonoscopy and abdominal ultrasonography. Several laboratory parameters and chest X-ray in all patients were assessed. All health care workers in procedure room were also tested with rapid antigen a week after the procedures. The study showed that laboratory parameters and chest X-ray were relatively normal for all patients. All procedures were conducted safely and leave no new COVID-19 case amongst health care workers. In conclusion, rapid test can be used in hospitals with limited resources, although RT-PCR test is still needed to confirm COVID-19 cases. Safe GI procedures can still be performed in limited resource hospital, although the possibility for disease transmission is still high. A modified guideline is needed in these hospitals in order to conduct safe GI procedures.
\end{abstract}

Keywords: COVID-19, gastrointestinal, rapid test, $R T-P C R$

\section{INTRODUCTION}

The world and medical community have experienced a huge viral pandemic since months ago named Corona Virus Disease 2019 (COVID19). All medical specialties and procedures are affected by the pandemic, including gastrointestinal (GI) procedures. The pandemic, caused by severe acute respiratory syndrome coronavirus 2 (SARSCoV-2), affects and changes gastrointestinal procedures' daily practice, both diagnostic and therapeutic [1]. Health care workers (HCWs) are at increased risk for COVID-19 because all GI procedures is a high-risk for disease transmission and maybe a potential route for COVID-19 [2], [3]. Considering that some GI procedures are aerosolgenerating and performed in close and prolonged contact with the patient, GI procedures must be considered as a very high risk of contagion [4].

International gastroenterology and gastrointestinal endoscopy societies have published guidelines and position statements focusing on endoscopy in the era of COVID-19 [1]. Recommendations have been changing rapidly and need to be updated regularly because the world faces sustained community transmission of COVID19 worldwide [2]. Performance of routine (elective) endoscopy is temporarily suspended a few months since the pandemic, and only emergency endoscopy is allowed in many countries, including Indonesia [3], [5]. The general strategy expressed by some national gastroenterology societies is to reduce contact with health-care settings and thus possible exposure to COVID-19. Therefore all non-essential GI procedures, including endoscopy and colonoscopy for cancer screening have been canceled, as these represent a risk for both patients and HCWs [6], [7]. Therefore, before conducting diagnostic or therapeutic GI procedures in patients with GI problems, the risks and benefits of performing the procedures should be considered. Firstly, it is important to ensure that the procedure is necessary and urgent. Candidate patients should be carefully selected, weighing transmission risks for other patients and HCWs [5].

All guidelines and recommendations suggest COVID-19 nucleic acid reverse transcriptase polymerase chain reaction (RT-PCR) testing must be done before gastrointestinal procedures within 3 days before endoscopy, although combination of Immunoglobulin (Ig) $\mathrm{M}$ and $\mathrm{G}$ antibodies against SARS-CoV-2 demonstrated $84.5 \%$ sensitivity and 91.6\% specificity. Additionally, some guidelines also suggest patients undergo 3-hour rapid testing on the day of gastrointestinal procedures [8], [9]. Patients are also asked to complete screening questionnaires [2], [10]. If no COVID-19 RT-PCR examination is available, all symptomatic/contact history patients are considered high risk patients for undergoing GI procedures [8]. 
Although RT-PCR is a gold standard, extremely sensitive, specific and often quantitative detection of the SARS-CoV-2 RNA, it has limitation as it is complex, expensive and slow to deliver as well as requires special expertise. A single RT-PCR test kit may cost over 100 USD, while setting up a diagnostic/processing lab requires more than 15,000 USD. These reasons make RT-PCR difficult to implement as regular diagnostic test in limited resource hospitals [11], [12]. Moreover, since lack of RT-PCR tests in Indonesia and the Government of Indonesia's regulation to use rapid antibodies for screening patients, we used rapid antibodies or antigen to screen patients before undergoing GI procedures [13]. The study aimed to analyze the use of antibody and antigen-based rapid tests to select patients for GI procedures and its impact on transmission of COVID-19 to the health care workers.

\section{METHODS}

The cross sectional study assessed total of 30 adult patients (20 males and 10 females) who met criteria for emergency and urgency endoscopies as previously described [14], underwent GI procedures such as abdominal ultrasonography (USG), endoscopy and colonoscopy during period of May to June 2020 in Zainoel Abidin Hospital (ZAH), a tertiary referred hospital in Province of Aceh, Indonesia. The procedures were conducted early during period of COVID-19 in Indonesia. Patients were screened with rapid test antigen or antibody against SARS-Cov-2 virus before underwent emergency GI procedures. Rapid antibody test SGTi-flex ${ }^{\circledR}$ COVID-19 IgM/IgG from Sugentech (Chungcheongbuk-do, South Korea) and BIOCREDIT ${ }^{\circledR}$ COVID-19 Antigen test by Rapigen (St Ingbert, Germany) were provided by government of Indonesia through ZAH and tested according manufacturer's procedures. Several parameters such as hemoglobin, total leukocytes, thrombocytes and neutrophil lymphocyte ratio (NLR) in all patients were assessed. Patients were also screened with chest X-rays. All HCWs who worked in the GI procedure room were tested for antigen against SARS-Cov-2 virus 1 week after GI procedures as screening procedure. All patients signed informed concern after getting explanation about objectives of the study. Ethical clearance was approved by the Research Ethics Commission of Faculty of Medicine Universitas Syiah Kuala and Zainoel Abidin Hospital, Banda Aceh, Indonesia, No. 37/EA/FL-RSUDZA/2020. Statistical analysis was performed with Graphpad ${ }^{\circledR}$ Prism ${ }^{\circledR} 5$ (San Diego, USA). Mean or median were used to analyze descriptive data.

\section{RESULTS AND DISCUSSIONS Baseline characteristic of patients}

We analyzed 30 patients before undergoing gastrointestinal (GI) procedures and determined baseline characteristics as shown in table 1. As shown in the table, two-thirds of patients are male with a median of age at 52.0 years old (32-87 years old). There were two main indications for emergency GI procedures; acute GI bleeding (hematemesis, melena and hematochezia) and right upper quadrant (RUQ) abdominal pain due to gall stones.

Table 1. Baseline characteristic of patients

\begin{tabular}{lc}
\hline Parameters & Results \\
\hline Sex (n, \%) & $20(66.6)$ \\
$\quad$ Male & $10(33.3)$ \\
$\quad$ Female & $52.0 \pm 14.9$ \\
Age (years), (median \pm SD) & \\
Indication of procedures (n, \%) & $27(90)$ \\
$\quad$ Acute GI bleeding & \\
$\quad$ RUQ Abdominal pain due to gall $3(10)$ \\
$\quad$ stone
\end{tabular}

Patients underwent GI procedures have nonreactive for antibodies and antigen against SARSCov-2, normal laboratory parameters and normal chest $X$-rays imaging

Since non-reactive for antibodies or antigen against SARS-Cov-2, normal laboratory parameters and normal chest X-ray were required to conduct GI procedures, antibodies, or antigen-based rapid tests were tested for all patients. Additionally, routine blood count was also measured for all patients as shown in Table 2. One patient was reactive for antigen SARS-Cov-2.

Table 2. Rapid test results and laboratory parameters

\begin{tabular}{lcc}
\hline \multicolumn{1}{c}{ Parameters } & Results \\
\hline IgM/IgG or antigen SARS-Cov-2 screening (reactive, \%) & $10.3 \pm 3.0$ & $239 \pm 28.5$ \\
\hline Hemoglobin (gr/dL (mean \pm SD) & $8.6 \pm 4.7$ \\
\hline Thrombocytes (x10 cells) (mean \pm SD) & $4,939 \pm 822.6$ \\
\hline Absolute Neutrophil Count (ANC) (median \pm SD) & $1,198 \pm 107.5$ \\
\hline Neutrophil Lymphocyte Ratio (NLR) (median \pm SD) & $3.8 \pm 0.9$ \\
\hline
\end{tabular}


Patient then underwent RT-PCR for confirming COVID-19 diagnosis. The RT-PCR could not confirm the diagnosis and patient then continued for GI procedures. As shown in the table 2, almost all laboratory parameters were normal for all patients, except for hemoglobin with mean and SD at $10.3 \pm 3.0$. Additionally, all patients have normal chest X-rays (data not shown).

\section{Most patients underwent urgency endoscopy}

As shown in figure 1, two thirds of total patients were undergoing endoscopy, 6 patients (20\%) colonoscopy and 3 patients $(10 \%)$ was abdominal USG. As shown in table 1 previously, acute upper GI bleeding indicated that patients underwent GI procedures (endoscopy).

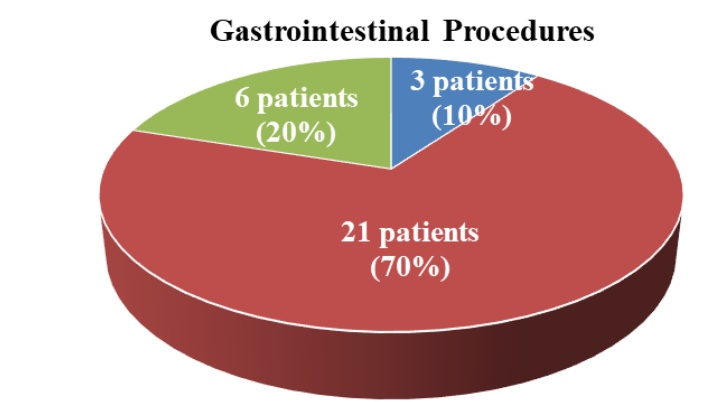

- Abdominal Ultrasonography $\quad$ Endoscopy $\quad$ Colonoscopy

Figure 1. Three gastrointestinal procedures that were conducted in Zainoel Abidin Hospital during early COVID-19 pandemic; endoscopy, colonoscopy and abdominal ultrasonography

\section{No new case of COVID-19 was found among health care worker of endoscopy facility}

We conducted rapid test antigen for all endoscopy facility personnel 1 week after all two months GI procedures. Total of 20 personnel (3 endoscopists, 2 anesthetists and 15 nurses) test and was showed non- reactive for antigen SARSCov-2 (data not shown). Their health condition was also monitored for duration of one month and showed no sign and symptoms for COVID-19 (data not shown).

The study showed for the first time experience of a limited resources hospital in Indonesia preparing patients for GI procedures during early period of COVID-19 pandemic, although a report from another endoscopy facility in Indonesia had showed the procedures before, during and after endoscopy. As far as we noticed, the report was the first report in Indonesia that present endoscopic procedures early during COVID-19 pandemic [3]. The report also divided endoscopy procedures into 3 groups based on urgency; urgent endoscopy, semi-urgent endoscopy and elective endoscopy. During early pandemic, all endoscopy facilities worldwide were recommended to conduct only urgent endoscopies or other GI procedures. As in the study, we only conducted emergency procedures such as acute GI bleeding or abdominal colic due to gall stones.

This recent study presents data on the initial screening of COVID-19 using rapid test early during pandemic in Indonesia. Laboratory analysis and chest $\mathrm{X}$-ray conducted after rapid test showed relatively normal parameters compared with COVID-19 confirmed case laboratories findings and chest X-rays [15]-[17]. This suggests that initial screening of prospective GI procedures patients is necessary, not only by assessing SARSCov-2 antibodies/antigens also assessing laboratory parameters and chest X-ray to exclude COVID-19 diagnosis. Although guidelines and recommendations suggested RT-PCR as standard diagnosis methods and if no RT-PCR examination is available [7], [18], all symptomatic/contact history patients are considered high risk patients, the limited resources hospital that has limited RTPCR tests should screen patients who will undergo GI procedures with these three different modalities; rapid test, laboratory parameters and chest X-ray. This can avoid transmission of COVID-19 from patients to HCWs, although RTPCR is still needed to confirm the diagnosis of COVID-19. However, since more laboratories can conduct the RT-PCR tests lately, especially in Indonesia, diagnosing COVID-19 become easier. Therefore, patients should be tested with RT-PCR first before undergo the GI procedures.

In addition, protection of medical workers is very important to during COVID-19 pandemic to conduct safe GI procedures as suggested by a report from Wuhan China as the epicenter of the world's first outbreak that ensures staff are working in a safe and clean environment is a critical step. There are several approaches can be done to prevent staff from COVID-19 transmission such as identifying and treating appropriately any member of staff that symptoms or signs for COVID-19 such as fever, fatigue or dry cough or who has had contacted with COVID19 infected patient, checking temperature of all HCWs everyday before entering the endoscopy facility, hand hygiene, protection at biosafety level 2 or 3 depends on procedures (such as biosafety level 3 for performing tracheal intubation, airway care, and sputum suction and endoscopic procedure) [4]. Most HCWs in endoscopy facility in our hospital have followed the suggestions therefore this was probably one of 
the reasons no new case found amongst HCWs in the study.

Finally, this study has several limitations, especially the number of patients that underwent GI procedures. This is due to lack of patients who came/were referred to the hospital because of pandemic. Moreover, we also limited patient who underwent GI procedures per week. A larger study population should be conducted to provide enhanced statistical reliability. In addition, we did not test for RT-PCR for HCWs who work in endoscopy facility.

\section{CONCLUSION}

Rapid test can be used in hospitals with limited resources, although RT-PCR tests are still needed to confirm Covid-19 cases. Safe gastrointestinal procedures can still be performed in a pandemic situation in limited resource hospital, although the possibility for disease transmission is still high. A modified guideline is needed in these hospitals in order to conduct safe gastrointestinal procedures.

\section{ACKNOWLEDGEMENT}

We would like to acknowledge the Ministry of Education and Culture of Indonesia.

\section{REFERENCES}

1. Gralnek, I. M., C. Hassan, and M. DinisRibeiro, 2020, COVID-19 and endoscopy: implications for healthcare and digestive cancer screening, Nat. Rev. Gastroenterol. Hepatol., 17(8), 444-446.

2. Elio C. C.F., Rodolfo C., Flavia F. F., Gustavo P., Hugo P., 2020, Gastrointestinal endoscopy during the COVID-19 pandemic: an updated review of guidelines and statements from international and national societies, Gastrointest. Endosc., 92(2), 440445.

3. H. Maulahela et al., 2020, Gastrointestinal Endoscopy in the Time of COVID-19 Pandemic: Current Guidelines and Experience From Indonesian Tertiary Endoscopy Centre, Indones. J. Gastroenterol. Hepatol. Dig. Endosc., 21(1), 45.

4. Y. Zhang, X. Zhang, L. Liu, H. Wang, and Q. Zhao, 2020, Suggestions for infection prevention and control in digestive endoscopy during current 2019-nCoV pneumonia outbreak in Wuhan, Hubei province, China., Endoscopy, 52(4), 312314.
5. Iacucci, M., Rosanna C., Nunzia L., Ren M., Remo P., Silvio D., Gursimran S K., Subrata G., Bo S., 2020, Endoscopy in inflammatory bowel diseases during the COVID-19 pandemic and post-pandemic period, Lancet Gastroenterol. Hepatol., 5(6), 598-606.

6. Mao, R., Jie L., Jun S., Subrata G., Liang-Ru Z., Hong Y., Kai-Chun W., Min-Hu C., 2020, Implications of COVID-19 for patients with pre-existing digestive diseases, Lancet Gastroenterol. Hepatol., 5(5), 425-427.

7. Bhandari, P., Pradeep B., Sunil D. J. E., Srisha H., Ian P., Sharmila S., Nigel T., Eleanor W., 2020, British Society of Gastroenterology (BSG) multi-society guidance on further recovery of endoscopy services during the post- pandemic phase of COVID-19. https://www.bsg.org.uk/.

8. Chiu PWY., Ng SC., Inoue H., Reddy DN., Ling Hu E., Cho JY., Ho LK., Hewett DG., Chiu HM., Rerknimitr R., Wang HP., Ho SH, Seo DW, Goh KL, Tajiri H, Kitano S, Chan FKL., 2020, Practice of endoscopy during COVID-19 pandemic: position statements of the Asian Pacific Society for Digestive Endoscopy (APSDE-COVID statements). Gut. Jun;69(6), 991-996.

9. Böger, B., Fachi, M. M., Vilhena, R. O., Cobre, A. de F., Tonin, F. S., \& Pontarolo, R., 2020, Systematic review with metaanalysis of the accuracy of diagnostic tests for COVID-19, J. Infect. Control, 1-9.

10. Han J, Wang Y, Zhu L, Cui Y, Li L, Zeng Z, Zhang S., 2020, Preventing the spread of COVID-19 in digestive endoscopy during the resuming period: meticulous execution of screening procedures. Gastrointest Endosc., 92(2):445-447.

11. A. Afzal, 2020, Molecular diagnostic technologies for COVID-19: Limitations and challenges, J. Adv. Res., 1-11.

12. K. Ramdas, A. Darzi, and S. Jain, 2020, 'Test, re-test, re-test': using inaccurate tests to greatly increase the accuracy of COVID19 testing, Nat. Med., 26(6), 810-811.

13. Djalante, R., Jonatan L., Davin S., Aruminingsih S., Mochamad I., Budi H., Choirul M., Muhammad S., S., Susanti D., Irina R., Lalu A G., Gusti A.K.S., Henny W, 2020, Review and analysis of current responses to COVID-19 in Indonesia: Period of January to March 2020, Prog. Disaster Sci., 6, 100091.

14. Mardani R., Ahmadi V. A., Zali F., Gholami A., Mousavi NSD., Kaghazian H., Kaviani M., Ahmadi N., 2020, Laboratory Parameters in Detection of COVID-19 Patients with Positive RT-PCR; a Diagnostic Accuracy Study. Arch Acad Emerg Med., 4;8(1), e43. 
15. Cozzi D, Albanesi M, Cavigli E, Moroni C, Bindi $\mathrm{A}$, Luvarà $\mathrm{S}$, Lucarini $\mathrm{S}$, Busoni $\mathrm{S}$, Mazzoni LN, Miele V., 2020 Chest X-ray in new Coronavirus Disease 2019 (COVID-19) infection: findings and correlation with clinical outcome. Radiol Med.,125(8):730737.

16. S. Minaee, R. Kafieh, M. Sonka, S. Yazdani, and G. Jamalipour S., 2020, Deep-COVID: Predicting COVID-19 from chest X-ray images using deep transfer learning, Med. Image Anal., 65, 101794.

17. Ang, T. L., 2020, Gastrointestinal endoscopy during COVID-19 pandemic, Gastroenterol. Hepatol., 35(5), 701-702. 